\title{
ADULT ONSET STILL'S DISEASE AS A CONSEQUENCE OF FULMINANT CHIKUNGUNYA FEVER: A CASE REPORT FROM BRAZIL'S NORTH REGION
}

Narottam Sócrates Garcia Chumpitaz (Universidade Federal de Roraima, Boa Vista, RR, Brasil), Mateus de Oliveria Lopes (Universidade Federal de Roraima, Boa Vista, RR, Brasil), Rodrigo dos Santos Silva (Universidade Federal de Roraima, Boa Vista, RR, Brasil), Bruno Leitão da Silva (Universidade Federal de Roraima, Boa Vista, RR, Brasil)

\section{BACKGROUND}

There are many arboviruses with rheumatologic symptoms, and the variable clinical settings are a major challenge for establishing a certainty diagnosis. We report a case of chronic bilateral polyarthralgia after chikungunya virus infection (CHIKV) in a state localized in the extreme north of Brazil.

\section{CASE REPORT}

ALSC, 48-year-old, male, natural and coming from São Miguel do Guamá- PA, public servant, previously healthy, admitted in June 2017 to the rheumatology service in the private network in the municipality of Boa Vista- RR, with fever complaints, myalgia, skin rash and symmetric bilateral polyarthritis for about three months.

On the occasion, laboratory tests were requested, where they presented the following results: Leukocytes 28,400 $\mathrm{LL}$, Neutrophils 89.5\%, Hb 10.6 g/dl, HSV 22 mm, PCR 96 mg/L, FR 16 IU/ml, negative VDRL. Serology for Chikungunya positive (IgM). Other serologies were negative from blood cultures.

Nonsteroidal anti-inflammatory drugs (NSAIDs) were started for acute treatment and after four weeks, the patient was admitted to the tertiary hospital of the state with a fair general health condition, macular lesions in proximal segments associated with fever and polyarthritis. Laboratory abnormalities: leukocytosis $(39,820 \mu \mathrm{L})$, hyperferritinemia $2,301 \mathrm{ng} / \mathrm{dl}$.

He was conducted for bone marrow biopsy. There was evidence of increased hematopoietic activity and cutaneous biopsy with no neoplastic infiltration. His CD4 and CD8 T lymphocytes were normal, other serologies requested were also negative. The laboratory presented FAN HEp-2 1/160 in a dotted reticular pattern. On physical examination, had erythematous eruption, fever, and polyarthritis.

The patient remained hospitalized for eight weeks. Observing the clinical-laboratory analysis, the most probable diagnosis was Still's Disease of the Adult. Pharmacological treatment with prednisolone with weaning schedule was instituted and Enbrel, Methotrexate and folic acid were also administered.

After three months, due to the maintenance of symptoms, the exchange of medication was performed for Tocilizumab associated with Leflunomide and Arcoxia, which had a good therapeutic response with significant clinical improvement.

\section{CONCLUSION}

Chikungunya virus infection is associated with the development of autoimmune, such as Still's disease, reported in the last decades. The Yamaguchi criteria are the most sensitive score for diagnosis (93.5\%). The inclusion of serum ferritin by a French group increased the specificity of the diagnosis (98.5\%).

Despite its non-specific presentation and absence of specific biomarkers, Still's disease should be included as a differential diagnosis in patients with fever of unknown-specific origin, musculoskeletal symptoms, and macular rash when other diagnoses are excluded. 